\title{
Ionotropic Receptors (IRs): Chemosensory ionotropic glutamate receptors in Drosophila and beyond
}

\author{
Raphael Rytz ${ }^{1}$, Vincent Croset ${ }^{1}$, Richard Benton* \\ Center for Integrative Genomics, Faculty of Biology and Medicine, University of Lausanne, CH-1015 Lausanne, Switzerland
}

\section{A R T I C L E I N F O}

\section{Article history:}

Received 15 November 2012

Received in revised form

30 January 2013

Accepted 13 February 2013

\section{Keywords:}

Olfaction

Gustation

Chemosensation

Ionotropic glutamate receptor

Drosophila melanogaster

Evolution

\begin{abstract}
A B S T R A C T
Ionotropic Receptors (IRs) are a recently characterized family of olfactory receptors in the fruit fly, Drosophila melanogaster. IRs are not related to insect Odorant Receptors (ORs), but rather have evolved from ionotropic glutamate receptors (iGluRs), a conserved family of synaptic ligand-gated ion channels. Here, we review the expression and function of IRs in Drosophila, highlighting similarities and differences with iGluRs. We also briefly describe the organization of the neuronal circuits in which IRs function, comparing and contrasting them with the sensory pathways expressing ORs. Finally, we summarize the bioinformatic identification and initial characterization of IRs in other species, which imply an evolutionarily conserved role for these receptors in chemosensation in insects and other protostomes.
\end{abstract}

(C) 2013 Elsevier Ltd. All rights reserved.

\section{Introduction}

In 1999, the discovery of Odorant Receptor (OR) genes in the fruit fly, Drosophila melanogaster, allowed definition of the molecular logic of insect olfactory system organization, permitted the development of genetic tools to visualize and manipulate specific olfactory pathways to determine how odors are encoded to evoke behavior, and founded comparative molecular evolutionary studies of the olfactory system across insects (Benton, 2007; Hansson and Stensmyr, 2011; Masse et al., 2009; Su et al., 2009; Vosshall and Stocker, 2007).

During the following decade, comprehensive anatomical and functional maps of Olfactory Sensory Neurons (OSNs) in both the peripheral sensory organs (the third antennal segment [hereafter, antenna] and maxillary palp) and the primary olfactory center in the brain (the antennal lobe) of Drosophila (Su et al., 2009; Vosshall and Stocker, 2007), revealed a large number of antennal neurons that do not express OR genes, or the related Gustatory Receptor (GR) genes (Montell, 2009; Vosshall and Stocker, 2007), implying the existence of another family of insect olfactory receptors. In 2009, through a bioinformatic and expression screen for novel olfactory genes (Benton et al., 2007), a large and highly

\footnotetext{
* Corresponding author. Tel.: +4121692 3932; fax: +4121692 3965. E-mail address: Richard.Benton@unil.ch (R. Benton)

1 These authors contributed equally to this work.
}

divergent family of ionotropic glutamate receptor (iGluR)-related genes, named Ionotropic Receptors (IRs), was proposed as the "missing" receptor repertoire (Benton et al., 2009).

In the past four years, characterization of Drosophila IRs, the neuronal circuits in which they function, and their homologs in other species, have revealed them to be an important and ancient repertoire of chemosensory receptors. Here, we synthesize these studies to provide a view of the current knowledge and open questions on the IRs.

\section{IR expression in the Drosophila olfactory system}

The Drosophila antenna is covered with porous sensory hairs, or sensilla, of three morphological classes - basiconic, trichoid and coeloconic - which house the ciliated dendritic endings of 1-4 OSNs (Fig. 1A). All basiconic and trichoid OSNs (as well as all maxillary palp OSNs) express OR genes, with the exception of the GR21a/GR63a $\mathrm{CO}_{2}$-sensing neurons (Couto et al., 2005; Fishilevich and Vosshall, 2005; Jones et al., 2007; Kwon et al., 2007; Su et al., 2009; Vosshall and Stocker, 2007). By contrast, antennal coeloconic ("ac") sensilla neurons do not express ORs, with one exception (OR35a) (Couto et al., 2005; Yao et al., 2005). There are two other sensory structures on the antenna: the arista, a feather-like projection (Foelix et al., 1989), and the sacculus, a multichambered "pit" (Shanbhag et al., 1995), whose neurons express neither ORs nor GRs. 
A
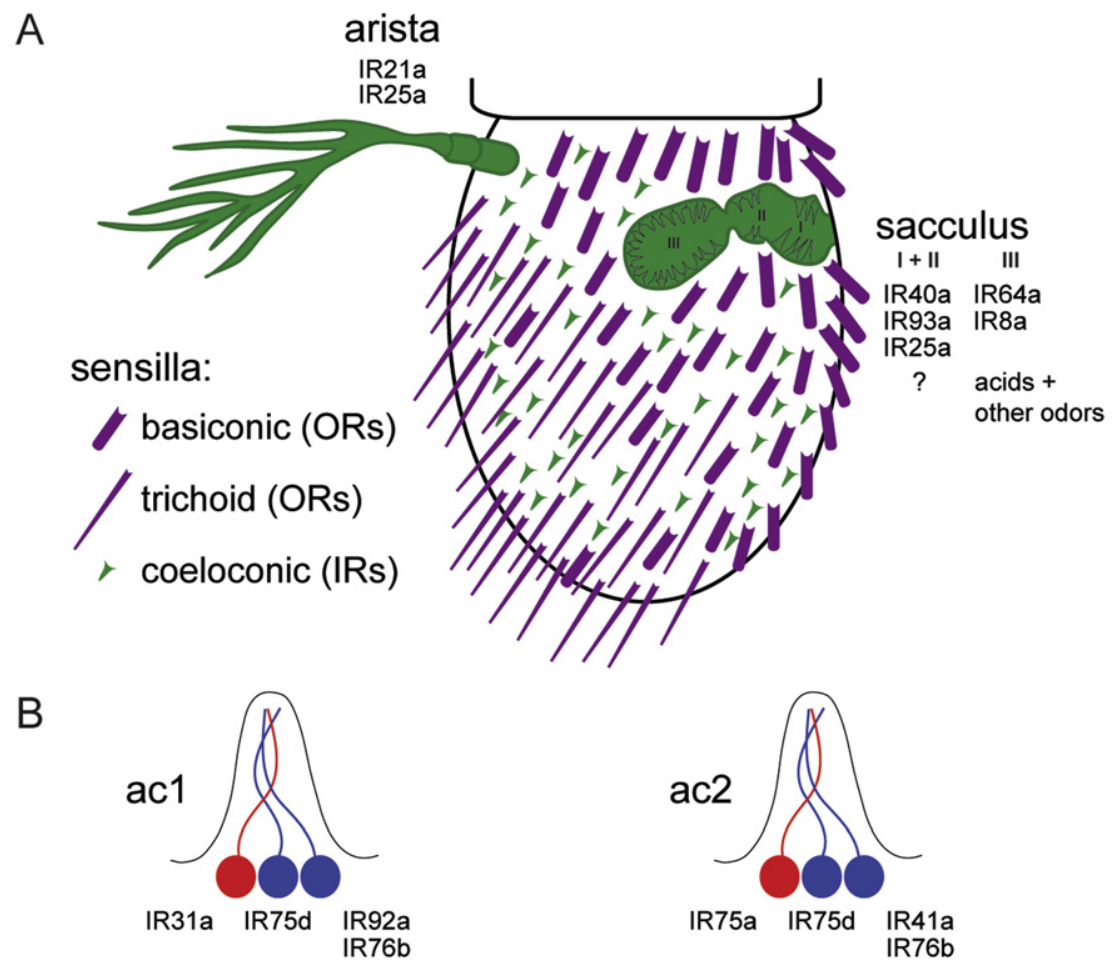

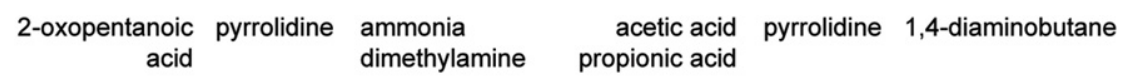

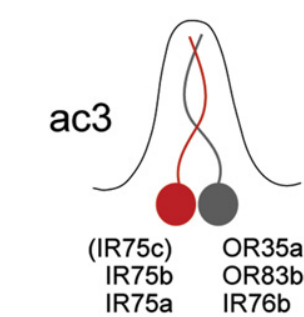

butyric acid many odors butyraldehyde (OR35apropionic acid dependent)

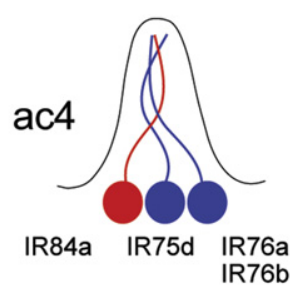

phenylacetic acid pyrrolidine phenylethylamine

IR8a co-receptor dependent

IR25a co-receptor dependent

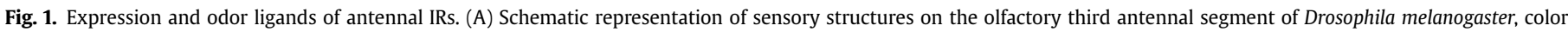

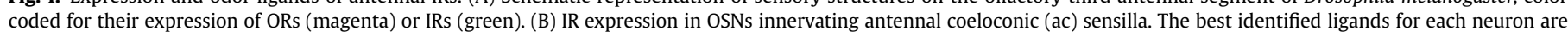

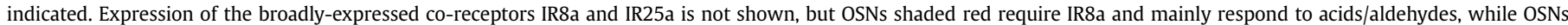
shaded blue require IR25a and mainly respond to amines.

Genomic analysis has identified 66 IR genes (including 9 putative pseudogenes) in D. melanogaster (Benton et al., 2009; Croset et al., 2010). Comprehensive expression analysis of these genes by RT-PCR, fluorescence RNA in situ hybridization and/or using transgenic reporters has shown that 16 of these are expressed in the antenna. Ten of these IRs are expressed in selective subsets of coeloconic sensilla OSNs (Benton et al., 2009; Croset et al., 2010), either uniquely, or co-expressed with 1-2 other IRs (Fig. 1B). Pioneering electrophysiological characterization of odor evokedresponses of coeloconic sensilla OSNs (see Section 3) defined the existence of four sensilla classes (ac1-ac4) with different response profiles and distinct but overlapping distributions on the surface of the antenna (Yao et al., 2005). The spatial map yielded by expression analysis of IR genes corresponded closely with the spatial distribution of the physiologically defined coeloconic classes (Yao et al., 2005), providing a compelling, though indirect, line of evidence that the receptors define the odor response profiles of these neurons (Benton et al., 2009). One neuronal class in ac3 sensilla co-expresses OR35a and IR76b, but the characterized odor responses in these neurons depend solely on the OR gene (Benton et al., 2009; Yao et al., 2005).

Two IRs, IR8a and IR25a, are expressed - at somewhat heterogeneous levels - in many of the coeloconic OSNs (Benton et al., 2009). Consistent with this broad expression, these receptors appear to function as co-receptors with several different, selectively expressed IRs (see Section 4) (Abuin et al., 2011; Benton et al., 2009). IR25a is additionally expressed, more weakly, in basiconic and trichoid OSNs, but its function in these cells, if any, is unclear (Benton et al., 2009).

The four remaining antennal IRs - IR21a, IR40a, IR64a and IR93a - are not found in coeloconic sensilla. IR21a is expressed, together with IR25a, in aristal neurons (Benton et al., 2009) 
(Fig. 1A). The arista contains thermosensory and not olfactory neurons, but the heat-sensing function is thought to be mediated by TRP channels (Foelix et al., 1989; Gallio et al., 2011) and the role of these IRs is unknown. The other IRs are expressed in sacculus neurons: IR40a, IR93a (and IR25a) in chambers I and II, nearest the opening on the antennal surface, and IR64a (together with IR8a) in neurons in the deepest chamber III (Fig. 1A). A morphological study of the sacculus has suggested that this structure houses olfactory, hygrosensory and thermosensory neurons (Shanbhag et al., 1995). IR64a/IR8a-expressing neurons are indeed olfactory (see Section 3) (Ai et al., 2010), while the function of IR40a/IR93a/IR25a neurons remains uncharacterized.

The expression of IRs in precise subpopulations of antennal neurons, together with a broadly expressed co-receptor(s) is analogous to the selective expression of ligand-specific ORs with their ubiquitous co-receptor ORCO (Benton et al., 2006; Larsson et al., 2004; Su et al., 2009; Vosshall and Stocker, 2007). Initial support that IRs form heteromeric complexes containing ligand-specific subunits and co-receptor subunits has now come from functional studies of these proteins (see Section 4). However, the observation that up to 5 IR genes are expressed in distinct, but often overlapping, combinations suggests a more complex logic for how IRs could function together to form olfactory receptors, perhaps similar to heterotetrameric iGluRs (Mayer, 2011).

Little is known about how IR expression is controlled. Unlike mammalian ORs (but like insect ORs), there is no evidence from loss-of-function or mis-expression studies that IRs are expressed monoallelically or that they convey negative feedback signals to inhibit expression of other IRs (Abuin et al., 2011; Benton et al., 2009; Fuss and Ray, 2009; Grosjean et al., 2011; Silbering et al., 2011). Transgenic reporter analysis has demonstrated that the transcriptional regulatory elements for most IRs are contained within fairly short regions of DNA (a few 100-1000 bp) upstream of the coding sequence (Ai et al., 2010; Benton et al., 2009; Silbering et al., 2011). IR expression may therefore be determined by the association of specific combinations of transcription factors to these sequences.

\section{Odor-response properties of IR-expressing neurons}

Odor ligands for IR-expressing OSNs have been characterized by screening panels of monomolecular odors by extracellular electrophysiological recordings in coeloconic sensilla (Silbering et al., 2011; Yao et al., 2005). Although this technique is relatively rapid, reliable spike-sorting of the 2 or 3 neurons housed in individual sensilla is difficult due to their similar spike amplitudes. Optical imaging of odor-evoked responses of specific IR OSN populations in the antennal lobe, by using IR promoters to express the calcium indicator GCaMP, has therefore proven useful to unambiguously assign responses to individual IR neuron classes (Ai et al., 2010; Silbering et al., 2012, 2011). This latter technique has also been important to examine responses of sacculus neurons, which are inaccessible to peripheral electrophysiological recordings (Ai et al., 2010).

Coeloconic OSNs have a spontaneous baseline activity that is dependent upon IR function (Grosjean et al., 2011; Yao et al., 2005). Firing frequency increases when the neuron is presented with an IR odor agonist, while it is decreased by inhibitory odors. Robust agonist-evoked neuronal responses are extremely sparse in coeloconic IR neurons: only 19 out of 168 chemically diverse odors tested produce increases in firing frequency of $>50$ spikes/s (about $25 \%$ of the maximum neuronal responses observed) (Silbering et al., 2011; Yao et al., 2005). Almost all of these agonists are amines, carboxylic acids or aldehydes (Fig. 1B). Although odor antagonists are less well-characterized, a few have been identified for certain IR neurons, and many of these are amines (Silbering et al., 2011; Yao et al.,
2005). Additionally, in ac1 and ac2 sensilla, the neuronal firing rate increases and decreases with increasing and decreasing humidity (Yao et al., 2005), but it is unclear whether these hygrosensory responses are specific to particular neurons, and whether they require IRs (Liu et al., 2007). In the sacculus, IR64a-expressing neurons comprise, unusually, two physiologically-distinct subpopulations: one responds selectively to all acidic stimuli (these neurons may be directly or indirectly activated by protons $\left[\mathrm{H}^{+}\right]$), while the other has broader responses to both acidic and non-acidic odors (Ai et al., 2010). What distinguishes these subpopulations molecularly is unknown.

Comparison of the olfactory responses of IR neurons with those of ORs has revealed several differences. First, IR neurons are generally more narrowly-tuned and less sensitive than OR neurons (Getahun et al., 2012; Silbering et al., 2011; Yao et al., 2005). Second, IR neurons are slower to respond but adapt less quickly (Getahun et al., 2012). Third, and most importantly, IRs and ORs detect distinct classes of odors (Silbering et al., 2011; Yao et al., 2005), with the strongest IR ligands recognized only weakly or not at all by ORs, and the strongest OR ligands (predominantly esters, alcohols and ketones (de Bruyne et al., 2001; Hallem and Carlson, 2006)) not stimulating any IR neurons. There are a few exceptions to these patterns (Silbering et al., 2011), and further screens, in particular those using natural, complex odor sources, will undoubtedly identify more potent ligands for IRs (and ORs). Nevertheless, the present observation of the largely complementary odor-sensing functions of IRs and ORs could explain the co-existence of two distinct repertoires within the Drosophila olfactory system.

\section{Molecular mechanism of IR function}

The homology of IRs to iGluRs has facilitated dissection of the mechanism by which these olfactory receptors localize to OSN sensory cilia, recognize odors and produce neuronal depolarization, because of the deep molecular understanding of these synaptic ligand-gated ion channels (Gereau and Swanson, 2008; Mayer, 2011). Like iGluRs, IRs contain a predicted extracellular N-terminus, a bipartite ligand-binding domain (LBD), whose two lobes (S1 and S2) are separated by an ion channel domain, and a short cytoplasmic C-terminal region (Fig. 2A). iGluRs also have a large extracellular amino-terminal domain (ATD) involved in channel assembly and binding of co-factors. A homologous domain is present in IR8a and IR25a, but it is highly divergent in the aristal and sacculus IRs (IR21a, IR40a, IR64a and IR93a), and apparently absent in other odor-specific IRs (Fig. 2A). Despite a similar domain organization to iGluRs, homology of IRs and iGluRs is low ( $<34 \%$ amino acid sequence identity), particularly within the LBD (Croset et al., 2010). Within the Drosophila IR repertoire, sequence homology ranges from 10 to $70 \%$, strongly suggesting functional diversity (Benton et al., 2009).

Demonstration that IRs are necessary and sufficient for odorevoked neuronal responses came from loss- and gain-of-function studies. For example, mutations in the ac4 phenylacetic acid/phenylacetaldehyde receptor IR84a or the sacculus receptor IR64a selectively abolish olfactory responses in their corresponding neurons (Ai et al., 2010; Grosjean et al., 2011). By contrast, mutations in the broadly-expressed IR8a and IR25a eliminate odorevoked responses in multiple, distinct classes of IR-expressing OSNs, suggesting that these receptors function as co-receptors with different sets of IRs (Abuin et al., 2011). Indeed, expression of IR84a in OR22a neurons (in basiconic sensilla) or in Xenopus oocytes, was sufficient to confer responses to phenylacetaldehyde only when co-expressed with IR8a. Co-expression of IR8a with a different receptor, IR75a, conferred responses to a different ligand, propionic acid (Abuin et al., 2011). 
A

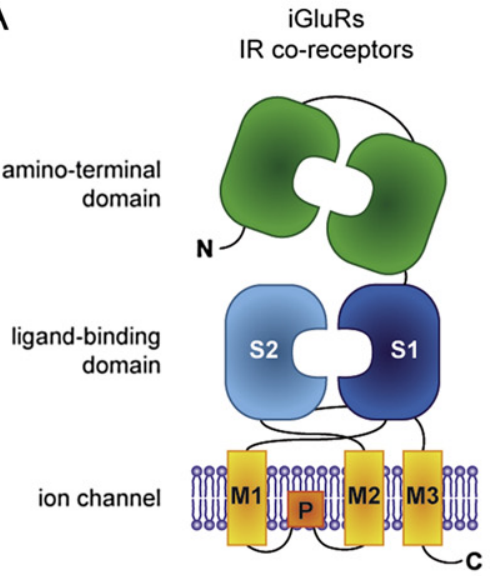

odor-specific IRs

B

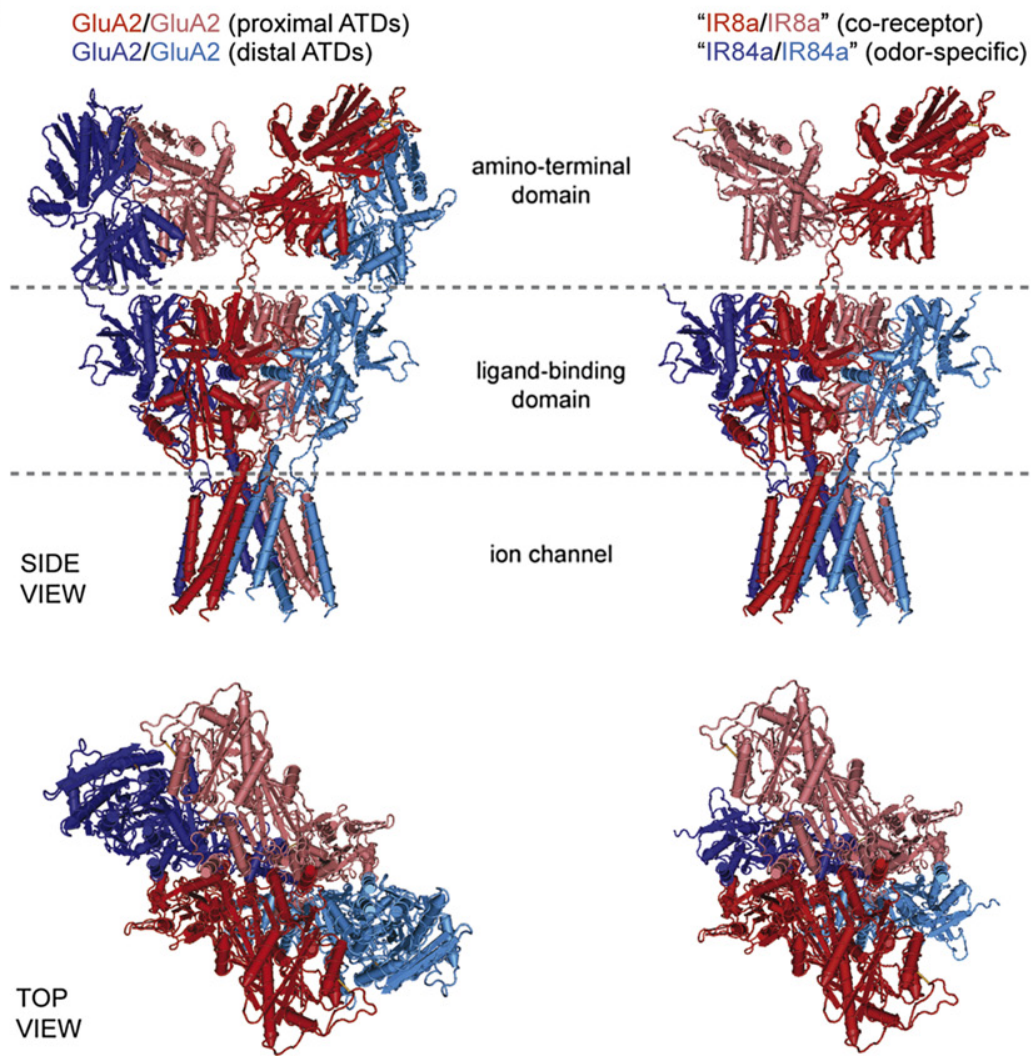


which IR84a (or other odor-specific IRs that lack ATDs, such as IR75a) can "insert" (Fig. 2B).

Beyond these speculations, it remains unclear whether the stoichiometry proposed for the IR84a/IR8a receptor is generalizable to all types of IR complex. For example, reconstitution of the ac4 phenylethylamine-sensing receptor requires three receptors: the presumed odor-specific IR76a, the broadly-expressed IR25a coreceptor, as well as IR76b, a receptor which is co-expressed with other IRs in ac1, ac2 as well as ac4 (Fig. 1B), and which may act as a second type of co-receptor (Abuin et al., 2011).

The oocyte expression system has also allowed demonstration of the ion conducting properties of IRs. Study of IR84a/IR8a and IR75a/IR8a showed that odor-evoked currents are carried preferentially by monovalent cations $\left(\mathrm{Na}^{+}\right.$and $\left.\mathrm{K}^{+}\right)$in both complexes, but that IR84a/IR8a is also slightly permeable to $\mathrm{Ca}^{2+}$. Interestingly, this $\mathrm{Ca}^{2+}$-permeability appears to depend upon a glutamine residue in the IR84a channel pore region; this residue (which is absent in IR75a) is also a major determinant of $\mathrm{Ca}^{2+}$-permeability in iGluRs (Liu and Zukin, 2007).

A key property of IRs is their specific targeting to the ciliated dendrites of OSNs (Benton et al., 2009). The correct localization of IRs probably depends upon complex formation as mutation of the IR8a co-receptor abolishes localization of IR84a and IR64a; reciprocally, IR8a localization is abolished or severely impaired in the absence of a partner odor-specific receptor (Abuin et al., 2011). Trafficking of iGluR complexes to postsynaptic membranes (topologically analogous to OSN cilia) is a highly regulated process that is dependent upon a number of quality-control steps ensuring correct subunit folding and complex assembly within the endomembrane system (Gereau and Swanson, 2008). Similar regulatory mechanisms may apply to IRs, but the small size of OSNs (soma diameter 2-3 $\mu \mathrm{m}$ (Shanbhag et al., 2000)) and their encasement within the antennal cuticle makes high-resolution in vivo imaging difficult. Initial structure-function analyses of IR8a and IR84a have implicated various domains in the correct localization of these proteins (Abuin et al., 2011); however, it was impossible to discriminate between a requirement in folding and a specific role in dendrite/cilia targeting. One intriguing observation is that a single amino acid mutation in the IR8a LBD (D724A), in a residue homologous to a glutamate-binding residue in iGluRs, abolishes cilia localization (Abuin et al., 2011). This hints that the co-receptor LBD may function in subcellular targeting rather than odor-recognition.

How IRs recognize their ligands remains an outstanding question. While their LBDs are highly divergent in overall primary sequence, secondary structure predictions suggest that they retain a similar overall fold to iGluRs (R.B., unpublished). Some IR LBDs retain one or more of the glutamate-binding residues of iGluR LBDs
(Abuin et al., 2011; Benton et al., 2009; Croset et al., 2010; Silbering et al., 2011). Indeed, mutation of an arginine residue in the IR84a LBD that is homologous to an arginine in iGluRs that contacts the $\alpha$-carboxyl group of the glutamate ligand completely abolishes odor responsiveness, while unaffecting receptor localization (Abuin et al., 2011). This result is consistent with a role for the IR LBD in odorrecognition, and suggestive of similarities in their ligand-binding mechanism with that of iGluRs. However, direct interactions between odors and IRs, the molecular basis for the observed specificity, and any ligand-induced conformational changes in the LBD (as observed in iGluRs (Mayer, 2011)) remain to be shown. These issues will most likely be best addressed by three-dimensional structural analysis of the IR LBD, a challenging but conceivable goal, given the success in atomic-resolution characterization of this domain in iGluRs (Mayer, 2011).

\section{Organization of IR olfactory circuits}

The identification of IR genes has also allowed the visualization of the olfactory circuits in which they are expressed, by using IR promoters to drive expression of neuroanatomical markers (Ai et al., 2010; Benton et al., 2009; Silbering et al., 2011). Like OR-expressing OSNs (Couto et al., 2005; Fishilevich and Vosshall, 2005), neurons expressing the same IR converge on a single glomerulus within the antennal lobe, to form a spatial map of sensory input (Fig. 3A). IR-expressing neurons in the sacculus display some exceptional anatomical properties. For example, IR64a OSNs project to two distinct glomeruli, DC4 and DP1m (Ai et al., 2010). It is not known whether individual IR64a neurons project to one or other, or both glomeruli. However, the former possibility seems more likely, as optical imaging has shown that these glomeruli have distinct odorresponse profiles (DC4 neurons are selective acid-sensors; DP1m are more broadly tuned), suggesting that IR64a OSNs comprise two physiologically and anatomically distinct subpopulations. IR40a/ IR93a-expressing neurons have a complex innervation pattern, comprising several dispersed subregions of the antennal lobe that do not clearly correspond to any previously defined glomerulus (Laissue et al., 1999). Whether this reflects a non-olfactory function for these neurons is unclear.

This analysis has essentially "completed" the antennal lobe molecular map of OSN innervations (Couto et al., 2005; Fishilevich and Vosshall, 2005; Silbering et al., 2011). This achievement allows consideration of the global organization of the IR olfactory subsystem compared with that of ORs, at the level of sensory input and at higher levels of the circuitry. Notably, while IR-expressing coeloconic OSNs and OR-expressing basiconic and trichoid OSNs are intermingled in the antenna (Fig. 1A), OR and IR glomeruli are
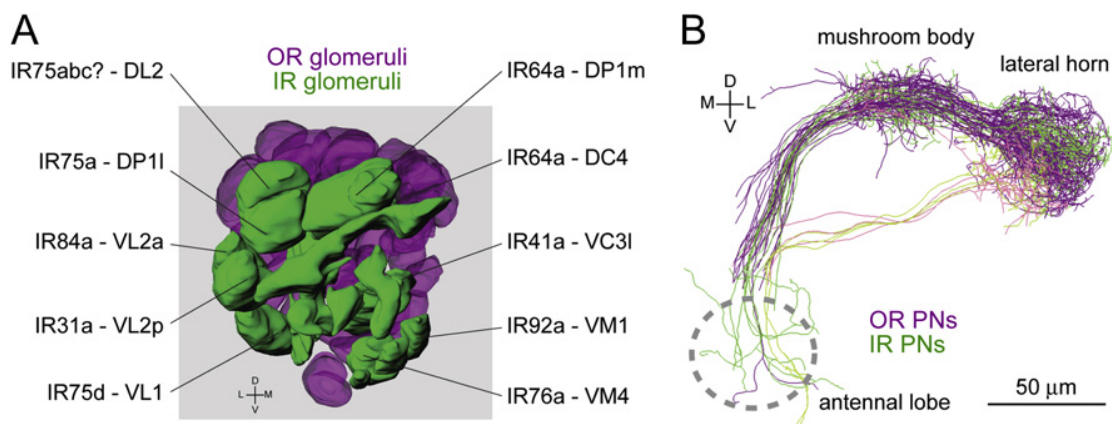

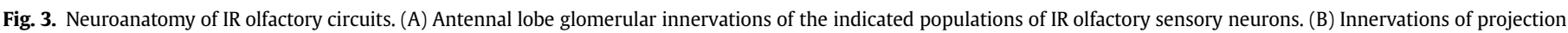

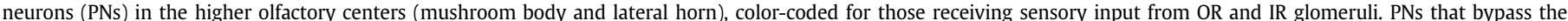

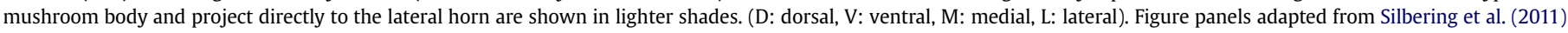
with permission. 
A

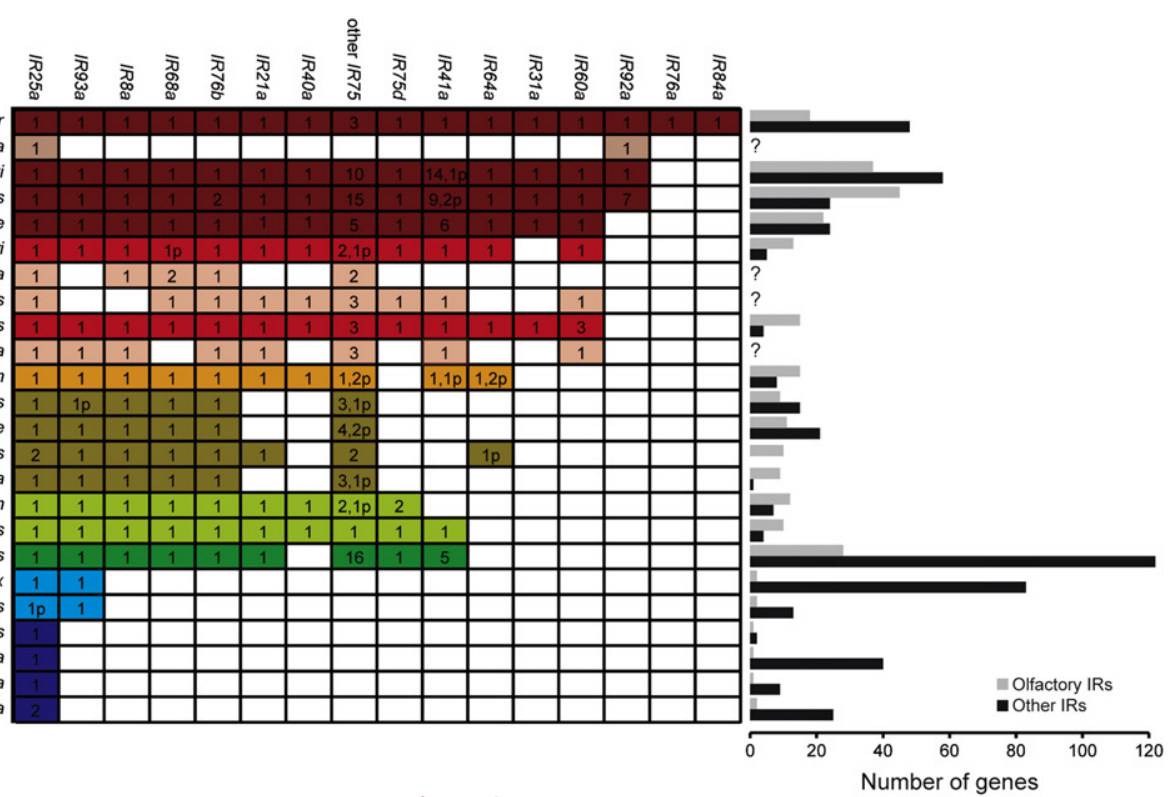

B

Diptera

Lepidoptera

Coleoptera

Hymenoptera

Hemiptera

Isoptera

Other arthropods

Other protostomes

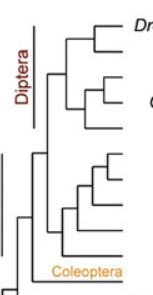
Rhagoletis pomonell Aedes aegypti Culex quinquefasciatus Anopheles gambiae

Bombyx mon

Manduca sexta

Spodoptera littoralis

Danaus plexippus

Cydia pomonella

Tribolium castaneum

gonomyrmex barbatus

Linepithema humile

Nasonia vitripennis
Apis mellifera

Acyrthosiphon pisum

Pediculus humanus

otermopsis nevadensis

Daphnia pulex

Ixodes scapularis

Capitella capitata

Aplysia californica

Lottia gigante

Number of genes

0

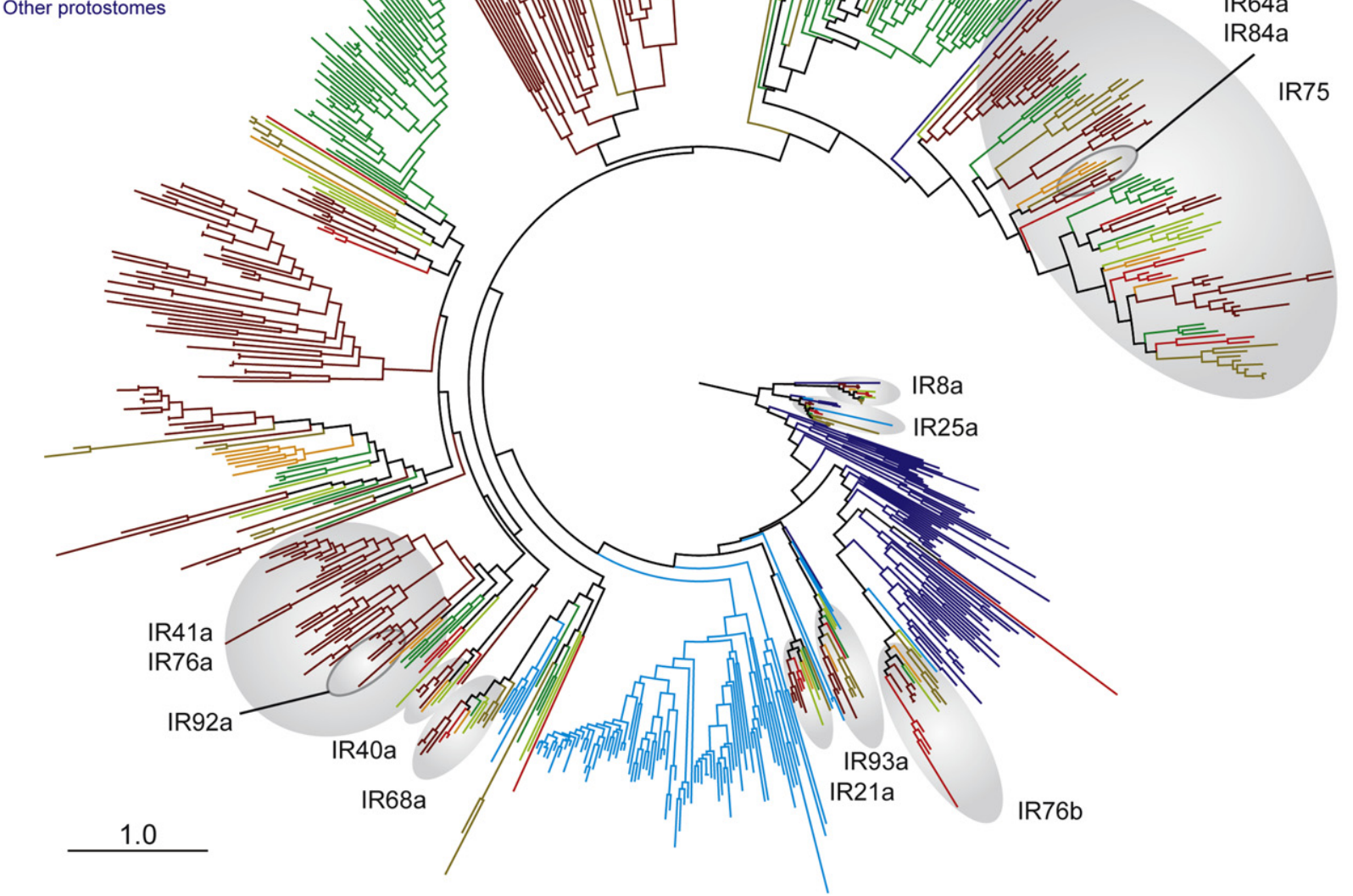

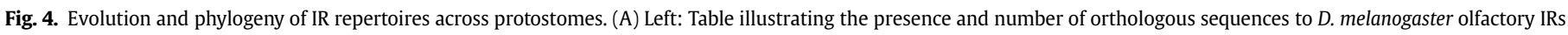

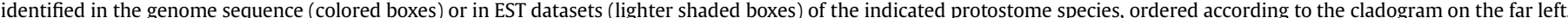

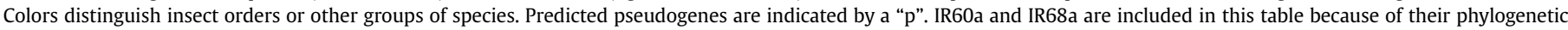

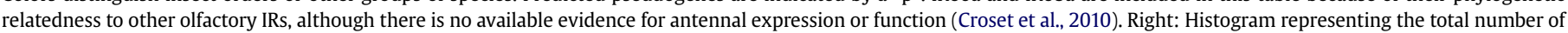

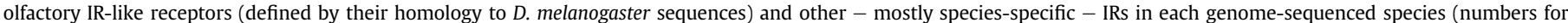

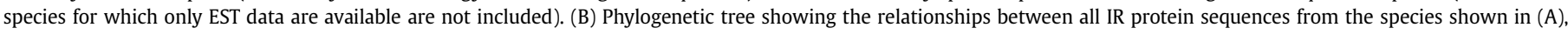


spatially segregated to anterior and posterior zones in the antennal lobe, respectively (Fig. 3A). The reason for this segregation is unknown, but may simply reflect a consequence of the earlier development and antennal lobe-innervation of IR OSNs (Jhaveri and Rodrigues, 2002; Silbering et al., 2011). Regardless, most antennal lobe local interneurons - whose processes are restricted to the lobe and synapse on both OSN input and projection neuron (PN) output pathways - do not discriminate between IR and OR glomeruli (Chou et al., 2010; Silbering et al., 2011). This anatomical observation suggests that substantial lateral excitatory and inhibitory interactions occur between OR and IR glomeruli.

Mapping of PNs receiving input from IR or OR glomeruli reveals that their axon termini are highly interdigitated within higher olfactory centers, the mushroom body and lateral horn (Fig. 3B) (Silbering et al., 2011). At present, very little is known about the organization and function of third-order neurons in the olfactory pathways. Nevertheless it is likely that while sensory input within these two olfactory subsystems is segregated in the antennal lobe, integration of sensory information occurs in the central brain to ultimately produce a co-ordinated behavioral response.

\section{Behaviors mediated by IR olfactory pathways}

Two IR pathways have been linked with particular odor-evoked behaviors in Drosophila. First, activity of the acid-sensing IR64a OSNs has been shown to be both necessary and sufficient to promote behavioral aversion (Ai et al., 2010). These neurons may reflect a sensory mechanism that allows flies to avoid unripe or over-fermented rotting fruit.

The second olfactory pathway is that expressing IR84a, which innervates the VL2a glomerulus (Grosjean et al., 2011). VL2a is one of three sexually-dimorphic glomeruli in the antennal lobe that express male-specific isoforms of fruitless, a master regulator of sexual behavior (Dickson, 2008). Consistent with this anatomical implication, mutation of IR84a reduces male courtship. Unexpectedly, the best ligands for IR84a, phenylacetaldehyde and phenylacetic acid, are not pheromones (that is, chemicals produced by other flies), but rather common odors found in the vegetal substrates (rotting fruits, flowers and other vegetal matter) where drosophilids feed and breed. These observations suggested a model in which the IR84a pathway detects environmental "aphrodisiacs" in food to promote sexual behavior of flies in a location where there are ample nutritional resources for their offspring (Grosjean et al., 2011).

Beyond these studies, it has been shown that flies that lack sensory input from all OR pathways (through mutation of the OR co-receptor ORCO (Benton et al., 2006; Larsson et al., 2004)) display odor-evoked responses to many chemically-diverse IR ligands (Silbering et al., 2011). Further genetic and chemical ecological analyses are required, however, to link these behaviors to particular IRs and to understand the natural context in which they are important.

\section{IR evolution in drosophilids}

While functional studies of IRs and their circuits have focused mostly on D. melanogaster, the wealth of genomic data now available has permitted investigation into the genetic conservation and divergence of these olfactory receptors during evolution at both relatively short and - as considered in Section 8 - long timescales. Within the Drosophila genus, the sequencing of many additional species that have diverse chemical ecology and behavior provide a particularly important resource to examine the genetics of olfactory evolution at a high phylogenetic resolution (Markow and O'Grady, 2007). Within the 12 species whose genomes were first available, IR repertoires range in size from 58 genes in Drosophila pseudoobscura, Drosophila virilis and Drosophila grimshawi to 69 in Drosophila simulans and Drosophila sechellia (Croset et al., 2010). The 16 antennal-expressed IRs (hereafter "olfactory IRs") are highly conserved across drosophilids, defining clearly distinct ortholog groups. Only a single predicted pseudogenization event, in D. sechellia IR75a, and only one gene duplication event, of Drosophila mojavensis IR75d, were identified in the olfactory IRs (Croset et al., 2010). These findings indicate that olfactory responses of IR neurons are probably largely conserved in drosophilids. $D$. sechellia, endemic to the Seychelles, and D. mojavensis, a cactophilic species endemic to the Sonoran desert of North America, are both specialists, with restricted food preferences, but it remains to be determined if the changes in their olfactory IR repertoires are linked to their particular ecological niches.

The 41 intact IRs whose expression is not detected in the D. melanogaster antenna display a more dynamic pattern of evolution within drosophilids. Many cases of gene loss, pseudogenization or duplication were identified (Croset et al., 2010). Moreover, the ratio of nonsynonymous to synonymous nucleotide substitutions (an indicator of the selection pressure acting on the coding sequence) is higher in these genes than in the olfactory IRs (Croset et al., 2010). The reduction or complete lack of introns in these genes compared with olfactory IRs suggests that they may have been born initially by retroposition of olfactory IRs (Croset et al., 2010). It is unclear if such an event happened only once or several times, because olfactory IRs do not form a monophyletic clade in phylogenetic trees (Fig.4C) (Croset et al., 2010). Many of the non-olfactory IR loci are organized in tandem arrays, indicating that expansion of this repertoire has probably subsequently been driven by non-allelic homologous recombination (Croset et al., 2010). The function of these receptors is still unknown, but transgenic reporters for three of them (IR7a, IR11a and IR100a) are expressed in larval and adult gustatory organs (Croset et al., 2010). It is likely that at least some of these IRs play a role in taste detection.

\section{IR evolution in insects and beyond}

Broader comparative genomic analyses of IR repertoires have provided insights into their evolutionary origin, expansion and diversification (Croset et al., 2010) (Fig. 4A-B). In contrast to the ORs, which are found only in insects, IRs are present in all protostome species examined, but not outside this clade (Fig. 4A). IRs may therefore have evolved in the last common protostome ancestor 550-850 million years ago. Because iGluR-like genes have a broader phylogenetic distribution - present in animals (Gereau and Swanson, 2008), plants (Lam et al., 1998; Michard et al., 2011) and prokaryotes (Chen et al., 1999; Chiu et al., 1999; Gereau

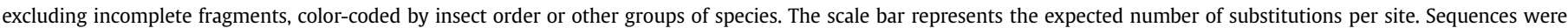

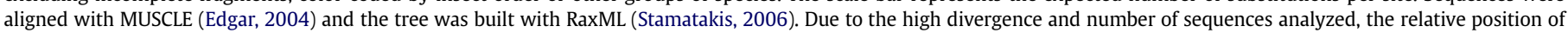

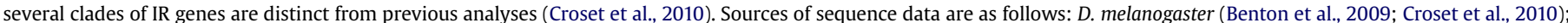

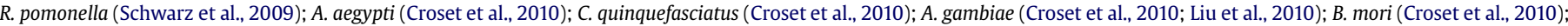

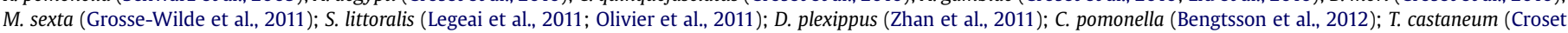

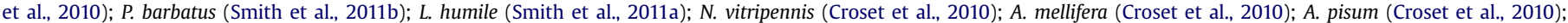

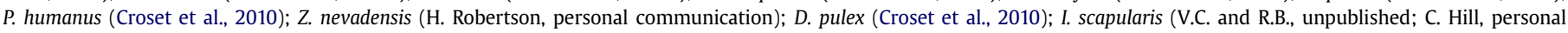
communication); C. elegans (Croset et al., 2010); C. capitata (Croset et al., 2010); A. californica (Croset et al., 2010); L. gigantea (Croset et al., 2010). 
and Swanson, 2008; Janovjak et al., 2010) - the most parsimonious model for the origin of IRs is that they derived from iGluRs. Indeed, phylogenetic and gene structure analyses indicate that IRs are likely to have shared a common ancestor with an AMPA or Kainate class of iGluR (Croset et al., 2010).

Analysis of the sequence relationships of IRs from different species with Drosophila IRs has allowed inference of the timing of origin of different receptors and how this relates to receptor function and the chemical ecology of different animals. One IR, IR25a, is conserved across protostomes (Fig. 4A-B) and is thus likely to be the ancestral IR. IR25a orthologs are expressed in olfactory and/or other chemosensory organs of many different insect species (Croset et al., 2010; Liu et al., 2010; Olivier et al., 2011; Pitts et al., 2011), lobsters (where the receptor is named OET-07) (Hollins et al., 2003; Stepanyan et al., 2004), Caenorhabditis elegans (where the receptor is named GLR-7) (Brockie et al., 2001) and Aplysia (Croset et al., 2010), suggesting a conserved chemosensory function for this receptor. Whether these orthologs act as co-receptors, as in Drosophila, remains to be determined. It is conceivable that the IR25a ancestor initially evolved as a sensory detector for external glutamate - analogous to the synaptic function of iGluRs - and that it only later acquired a co-receptor function after duplication and diversification of the IR repertoire.

Many other olfactory IRs in Drosophila have clear orthologs in most or all other insects (Fig. 4A-B) and these appear to be antennal-expressed (typically based on EST datasets or RT-PCR analysis) in several species, including mosquitoes (Liu et al., 2010; Pitts et al., 2011), honeybees (Croset et al., 2010) and moths (Bengtsson et al., 2012; Grosse-Wilde et al., 2011; Legeai et al., 2011; Olivier et al., 2011). This conservation stands in stark contrast to the ORs, where only the co-receptor ORCO is recognizable across insects (Jones et al., 2005), and suggests that the IR ligands are important olfactory cues for all insects. Acids and amines, for example, are key host-seeking signals in mosquitoes (van der Goes van Naters and Carlson, 2006), and it is highly likely that the Anopheles gambiae IRs (Liu et al., 2010) underlie the acid and amine responses in grooved peg sensilla OSNs in the adult antenna (Qiu et al., 2006). Consistently, a study of larval A. gambiae showed that small interfering RNA (siRNA) knock-down of the IR76b ortholog altered behavioral responses to butylamine (Liu et al., 2010).

Some olfactory IRs appear to have more restricted conservation, and these may underlie more species-specific chemosensory behaviors. IR84a, for example, is only found in drosophilids, and its role in regulating courtship behavior may be a unique strategy of fruit flies (Grosjean et al., 2011). There are several examples of species-specific expansions of particular olfactory IR clades, such as the IR75 subfamily, represented by just one member in the body louse, Pediculus humanus, 3 in drosophilids, but 15 in the mosquito Culex quinquefasciatus (Fig. 4A-B). Although the odor specificity of individual members of these large expansions is unknown, we suggest that such clades may underlie detection of closely related chemicals that need to be discriminated with high-resolution.

With a few exceptions (e.g. the IR7 clade and IR100a), the Drosophila IRs that are not expressed in the antenna have no closely related homologs outside drosophilids (Croset et al., 2010). Similarly, while many insects have a common set of presumed olfactory IRs, their repertoires are otherwise enormously variable in sequence and number across species, ranging from 1 in the honeybee Apis mellifera (Croset et al., 2010) to 122 in the termite Zootermopsis nevadensis ( $\mathrm{H}$. Robertson, personal communication). There are also several examples of lineage specific expansions of IR genes, for example, the IR317 subfamily in the ant Camponotus floridanus (Zhou et al., 2012), which might have species-specific functions. IR repertoire diversity further extends outside insects: beyond the conserved IR25a (as well as an IR93a ortholog in the waterflea Daphnia pulex and the tick Ixodes scapularis), there are no receptors with close homology to any insect receptors, and the families can number from as few as 3 in C. elegans to 85 in D. pulex (Fig. 4A) (Croset et al., 2010). Some chemosensory expression data are available in C. elegans, where they appear to be in the pharyngeal neurons (Brockie et al., 2001), which may contact ingested food, and in Aplysia sea slugs, where several are expressed in the rhinophore olfactory organ (Croset et al., 2010). However, the expression is unknown for the vast majority of IRs, and no ligands have been identified for any of them. Their functions, and the selection pressures driving their diversification, remain, therefore unclear.

\section{Conclusions and perspectives}

Since the discovery of IRs, we have learned much about their evolution, expression and function, and about the sensory circuits in which they act. Within insects, IRs appear to define an olfactory subsystem that exhibits many, though not all, of the organizational principles of the OR subsystem. The IR olfactory circuits therefore define a complementary model in which to address both developmental issues of, for example, olfactory receptor gene choice and OSN wiring, and neurobiological questions, including how odors are represented as spatio-temporal neural activity patterns in the brain and how these induce behavior. While progress has been made in several of these areas for the OR subsystem (Fuss and Ray, 2009; Jefferis and Hummel, 2006; Masse et al., 2009), it is not evident that the developmental and functional strategies are identical or even similar for the circuits expressing IRs.

IRs and ORs are clearly distinguished by their structure and their ligand specificity. The clear homology of IRs and iGluRs has helped to address molecular mechanisms by which odor recognition is converted into neuronal depolarization by these receptors - which are issues that still remain unclear for ORs (Nakagawa and Vosshall, 2009) - and further advancement in the future seems likely. Reciprocally, study of IRs may help illuminate previously unanticipated features of this widely conserved family of ligand-gated ion channel, such as the molecular basis and evolution of ligand binding specificity by the common fold of the bi-lobed LBD.

Within $D$. melanogaster, the olfactory IRs represent just a quarter of the entire repertoire, so definition of the site of expression and function of the remaining larger fraction represents a key area of interest. Intriguingly, one of these receptors, IR94b, was recently implicated in auditory system function (Senthilan et al., 2012), raising the possibility that IRs may also have non-chemosensory roles. Beyond Drosophila, large repertoires of IR sequences in molluscs, annelids, nematode worms, crustaceans and diverse insects remain to be characterized, and it will be exciting to see the functional conservation and divergence of this repertoire across these ecologically disparate species.

Finally, the IRs, and their circuits, offer an interesting model to understand fine-scale evolution of the nervous system (Ramdya and Benton, 2010). The revelation of a progressive expansion of the olfactory IR repertoire across protostomes (Fig. 4A) - whose resolution will certainly be increased with additional insect genomes, particularly from orthopteran species (Hansson and Stensmyr, 2011; Rebora et al., 2012) - invites comparative analyses both between individual receptors/circuits within a particular species, and between presumed conserved receptors in different species. These will advance our understanding of how olfactory receptor genes are duplicated and diversified, and segregated to new neural pathways to define unique odor-evoked behaviors. 


\section{Conflict of interest}

The authors declare no conflict of interest.

\section{Acknowledgments}

We thank Harald Janovjak, Richard Newcomb and Ana Silbering for discussions and comments on the manuscript. R.R. was supported by the Roche Research Foundation. V.C. was supported by a PhD Fellowship from the Boehringer Ingelheim Fonds. Research in R.B.'s laboratory is supported by the University of Lausanne, a European Research Council Starting Independent Researcher Grant and the Swiss National Science Foundation.

\section{References}

Abuin, L., Bargeton, B., Ulbrich, M.H., Isacoff, E.Y., Kellenberger, S., Benton, R., 2011 Functional architecture of olfactory ionotropic glutamate receptors. Neuron 69 , 44-60.

Ai, M., Min, S., Grosjean, Y., Leblanc, C., Bell, R., Benton, R., Suh, G.S., 2010. Acid sensing by the Drosophila olfactory system. Nature 468, 691-695.

Bengtsson, J.M., Trona, F., Montagne, N., Anfora, G., Ignell, R., Witzgall, P., JacquinJoly, E., 2012. Putative chemosensory receptors of the codling moth, Cydia pomonella, identified by antennal transcriptome analysis. PLoS One 7, e31620.

Benton, R., 2007. Sensitivity and specificity in Drosophila pheromone perception. Trends Neurosci. 30, 512-519.

Benton, R., Sachse, S., Michnick, S.W., Vosshall, L.B., 2006. Atypical membrane topology and heteromeric function of Drosophila odorant receptors in vivo. PLoS Biol. 4, e20.

Benton, R., Vannice, K.S., Gomez-Diaz, C., Vosshall, L.B., 2009. Variant ionotropic glutamate receptors as chemosensory receptors in Drosophila. Cell 136, 149-162.

Benton, R., Vannice, K.S., Vosshall, L.B., 2007. An essential role for a CD36-related receptor in pheromone detection in Drosophila. Nature 450, 289-293.

Brockie, P.J., Madsen, D.M., Zheng, Y., Mellem, J., Maricq, A.V., 2001. Differential expression of glutamate receptor subunits in the nervous system of Caenorhabditis elegans and their regulation by the homeodomain protein UNC-42. J. Neurosci. 21, 1510-1522.

Chen, G.Q., Cui, C., Mayer, M.L., Gouaux, E., 1999. Functional characterization of a potassium-selective prokaryotic glutamate receptor. Nature 402, 817-821.

Chiu, J., DeSalle, R., Lam, H.M., Meisel, L., Coruzzi, G., 1999. Molecular evolution of glutamate receptors: a primitive signaling mechanism that existed before plants and animals diverged. Mol. Biol. Evol. 16, 826-838.

Chou, Y.H., Spletter, M.L., Yaksi, E., Leong, J.C., Wilson, R.I., Luo, L., 2010. Diversity and wiring variability of olfactory local interneurons in the Drosophila antennal obe. Nat. Neurosci. 13, 439-449.

Couto, A., Alenius, M., Dickson, B.J., 2005. Molecular, anatomical, and functional organization of the Drosophila olfactory system. Curr. Biol. 15, 1535-1547.

Croset, V., Rytz, R., Cummins, S.F., Budd, A., Brawand, D., Kaessmann, H., Gibson, T.J., Benton, R., 2010. Ancient protostome origin of chemosensory ionotropic glutamate receptors and the evolution of insect taste and olfaction. PLoS Genet. 6, e1001064

de Bruyne, M., Foster, K., Carlson, J.R., 2001. Odor coding in the Drosophila antenna. Neuron 30, 537-552.

Dickson, B.J., 2008. Wired for sex: the neurobiology of Drosophila mating decisions Science 322, 904-909.

Edgar, R.C., 2004. MUSCLE: multiple sequence alignment with high accuracy and high throughput. Nucleic Acids Res. 32, 1792-1797.

Fishilevich, E., Vosshall, L.B., 2005. Genetic and functional subdivision of the Drosophila antennal lobe. Curr. Biol. 15, 1548-1553.

Foelix, R.F., Stocker, R.F., Steinbrecht, R.A., 1989. Fine structure of a sensory organ in the arista of Drosophila melanogaster and some other dipterans. Cell. Tissue Res. 258, 277-287.

Fuss, S.H., Ray, A. 2009. Mechanisms of odorant receptor gene choice in Drosophila and vertebrates. Mol. Cell. Neurosci. 41, 101-112.

Gallio, M., Ofstad, T.A., Macpherson, L.J., Wang, J.W., Zuker, C.S., 2011. The coding of temperature in the Drosophila brain. Cell 144,614-624.

Gereau, R.W., Swanson, G.T., 2008. The Glutamate Receptors. Humana Press, Totowa, N.J.

Getahun, M.N., Wicher, D., Hansson, B., Olsson, S.B., 2012. Temporal response dynamics of Drosophila olfactory sensory neurons depends on receptor type and response polarity. Front. Cell. Neurosci. 54. http://dx.doi.org/10.3389/fncel.2012.00054.

Grosjean, Y., Rytz, R., Farine, J.P., Abuin, L., Cortot, J., Jefferis, G.S., Benton, R., 2011. An olfactory receptor for food-derived odours promotes male courtship in Drosophila. Nature 478, 236-240.

Grosse-Wilde, E., Kuebler, L.S., Bucks, S., Vogel, H., Wicher, D., Hansson, B.S., 2011 Antennal transcriptome of Manduca sexta. Proc. Natl. Acad. Sci. U.S.A. 108 7449-7454.

Hallem, E.A., Carlson, J.R., 2006. Coding of odors by a receptor repertoire. Cell 125, $143-160$
Hansson, B.S., Stensmyr, M.C., 2011. Evolution of insect olfaction. Neuron 72, 698-711.

Hollins, B., Hardin, D., Gimelbrant, A.A., McClintock, T.S., 2003. Olfactory-enriched transcripts are cell-specific markers in the lobster olfactory organ. J. Comp. Neurol. 455, 125-138.

Janovjak, H., Szobota, S., Wyart, C., Trauner, D., Isacoff, E.Y., 2010. A light-gated, potassium-selective glutamate receptor for the optical inhibition of neuronal firing. Nat. Neurosci. 13, 1027-1032.

Jefferis, G.S., Hummel, T., 2006. Wiring specificity in the olfactory system. Semin. Cell. Dev. Biol. 17, 50-65.

Jhaveri, D., Rodrigues, V., 2002. Sensory neurons of the Atonal lineage pioneer the formation of glomeruli within the adult Drosophila olfactory lobe. Development 129, 1251-1260.

Jones, W.D., Cayirlioglu, P., Grunwald Kadow, I., Vosshall, L.B., 2007. Two chemosensory receptors together mediate carbon dioxide detection in Drosophila. Nature 445, 86-90.

Jones, W.D., Nguyen, T.A., Kloss, B., Lee, K.J., Vosshall, L.B., 2005. Functional conservation of an insect odorant receptor gene across 250 million years of evolution. Curr. Biol. 15, R119-R121.

Kwon, J.Y., Dahanukar, A., Weiss, L.A., Carlson, J.R., 2007. The molecular basis of $\mathrm{CO}_{2}$ reception in Drosophila. Proc. Natl. Acad. Sci. U.S.A. 104, 3574-3578.

Laissue, P.P., Reiter, C., Hiesinger, P.R., Halter, S., Fischbach, K.F., Stocker, R.F., 1999. Three-dimensional reconstruction of the antennal lobe in Drosophila melanogaster. J. Comp. Neurol. 405, 543-552.

Lam, H.M., Chiu, J., Hsieh, M.H., Meisel, L., Oliveira, I.C., Shin, M., Coruzzi, G., 1998. Glutamate-receptor genes in plants. Nature 396, 125-126.

Larsson, M.C., Domingos, A.I., Jones, W.D., Chiappe, M.E., Amrein, H., Vosshall, L.B. 2004. Or83b encodes a broadly expressed odorant receptor essential for Drosophila olfaction. Neuron 43, 703-714.

Legeai, F., Malpel, S., Montagne, N., Monsempes, C., Cousserans, F., Merlin, C., Francois, M.C., Maibeche-Coisne, M., Gavory, F., Poulain, J., Jacquin-Joly, E., 2011. An expressed sequence tag collection from the male antennae of the Noctuid moth Spodoptera littoralis: a resource for olfactory and pheromone detection research. BMC Genomics 12, 86

Liu, C., Pitts, RJ., Bohbot, J.D., Jones, P.L, Wang G, Zwiebel, LJ, 2010. Distinct olfactory signaling mechanisms in the malaria vector mosquito Anopheles gambiae. PLoS Biol. 8, e1000467.

Liu, L., Li, Y., Wang, R., Yin, C., Dong, Q., Hing, H., Kim, C., Welsh, M.J., 2007. Drosophila hygrosensation requires the TRP channels water witch and nanchung. Nature 450, 294-298.

Liu, S.J., Zukin, R.S., 2007. $\mathrm{Ca}^{2+}$-permeable AMPA receptors in synaptic plasticity and neuronal death. Trends Neurosci. 30, 126-134.

Markow, T.A., O'Grady, P.M., 2007. Drosophila biology in the genomic age. Genetics 177, 1269-1276.

Masse, N.Y., Turner, G.C., Jefferis, G.S., 2009. Olfactory information processing in Drosophila. Curr. Biol. 19, R700-R713.

Mayer, M.L., 2011. Emerging models of glutamate receptor ion channel structure and function. Structure 19, 1370-1380.

Michard, E., Lima, P.T., Borges, F., Silva, A.C., Portes, M.T., Carvalho, J.E., Gilliham, M. Liu, L.H., Obermeyer, G., Feijo, J.A., 2011. Glutamate receptor-like genes form $\mathrm{Ca} 2+$ channels in pollen tubes and are regulated by pistil D-serine. Science 332 , 434-437.

Montell, C., 2009. A taste of the Drosophila gustatory receptors. Curr. Opin. Neurobiol. 19, 345-353.

Nakagawa, T., Vosshall, L.B., 2009. Controversy and consensus: noncanonical signaling mechanisms in the insect olfactory system. Curr. Opin. Neurobiol. 19, 284-292.

Olivier, V., Monsempes, C., Francois, M.C., Poivet, E., Jacquin-Joly, E., 2011. Candidate chemosensory ionotropic receptors in a Lepidoptera. Insect Mol. Biol. 20, 189-199.

Pitts, R.J., Rinker, D.C., Jones, P.L., Rokas, A., Zwiebel, L.J., 2011. Transcriptome profiling of chemosensory appendages in the malaria vector Anopheles gambiae reveals tissue- and sex-specific signatures of odor coding. BMC Genomics 12, 271.

Qiu, Y.T., van Loon, J.J., Takken, W., Meijerink, J., Smid, H.M., 2006. Olfactory coding in antennal neurons of the malaria mosquito, Anopheles gambiae. Chem. Senses 31, 845-863.

Ramdya, P., Benton, R., 2010. Evolving olfactory systems on the fly. Trends Genet. 26, 307-316.

Rebora, M., Salerno, G., Piersanti, S., Dell'otto, A., Gaino, E., 2012. Olfaction in dragonflies: electrophysiological evidence. J. Insect Physiol. 58, 270-277.

Schwarz, D., Robertson, H.M., Feder, J.L., Varala, K., Hudson, M.E., Ragland, G.J., Hahn, D.A., Berlocher, S.H., 2009. Sympatric ecological speciation meets pyrosequencing: sampling the transcriptome of the apple maggot Rhagoletis pomonella. BMC Genomics 10, 633.

Senthilan, P.R., Piepenbrock, D., Ovezmyradov, G., Nadrowski, B., Bechstedt, S., Pauls, S., Winkler, M., Mobius, W., Howard, J., Gopfert, M.C., 2012. Drosophila auditory organ genes and genetic hearing defects. Cell 150, 1042-1054.

Shanbhag, S.R., Muller, B., Steinbrecht, R.A., 2000. Atlas of olfactory organs of Drosophila melanogaster 2. Internal organization and cellular architecture of olfactory sensilla. Arthropod Struct. Dev. 29, 211-229.

Shanbhag, S.R., Singh, K., Singh, R.N., 1995. Fine structure and primary sensory projections of sensilla located in the sacculus of the antenna of Drosophila melanogaster. Cell. Tissue Res. 282, 237-249.

Silbering, A.F., Bell, R., Galizia, C.G., Benton, R., 2012. Calcium imaging of odorevoked responses in the Drosophila antennal lobe. J. Vis. Exp. 61, pii: 2976, http://dx.doi.org/10.103791/2976. 
Silbering, A.F., Rytz, R., Grosjean, Y., Abuin, L., Ramdya, P., Jefferis, G.S., Benton, R., 2011. Complementary function and integrated wiring of the evolutionarily distinct Drosophila olfactory subsystems. J. Neurosci. 31, 13357-13375.

Smith, C.D., Zimin, A., Holt, C., Abouheif, E., Benton, R., Cash, E., Croset, V., Currie, C.R., Elhaik, E., Elsik, C.G., Fave, M.J., Fernandes, V., Gadau, J., Gibson, J.D., Graur, D., Grubbs, K.J., Hagen, D.E., Helmkampf, M., Holley, J.A., Hu, H., Viniegra, A.S., Johnson, B.R., Johnson, R.M., Khila, A., Kim, J.W., Laird, J., Mathis, K.A., Moeller, J.A., Munoz-Torres, M.C., Murphy, M.C., Nakamura, R., Nigam, S., Overson, R.P., Placek, J.E., Rajakumar, R., Reese, J.T., Robertson, H.M. Smith, C.R., Suarez, A.V., Suen, G., Suhr, E.L., Tao, S., Torres, C.W., van Wilgenburg, E., Viljakainen, L., Walden, K.K., Wild, A.L., Yandell, M., Yorke, J.A., Tsutsui, N.D., 2011a. Draft genome of the globally widespread and invasive Argentine ant (Linepithema humile). Proc. Natl. Acad. Sci. U.S.A. 108, 5673-5678.

Smith, C.R., Smith, C.D., Robertson, H.M., Helmkampf, M., Zimin, A., Yandell, M., Holt, C., Hu, H., Abouheif, E., Benton, R., Cash, E., Croset, V., Currie, C.R., Elhaik, E., Elsik, C.G., Fave, M.J., Fernandes, V., Gibson, J.D., Graur, D., Gronenberg, W., Grubbs, K.J., Hagen, D.E., Viniegra, A.S., Johnson, B.R., Johnson, R.M., Khila, A., Kim, J.W., Mathis, K.A., Munoz-Torres, M.C., Murphy, M.C., Mustard, J.A., Nakamura, R., Niehuis, O., Nigam, S., Overson, R.P., Placek, J.E., Rajakumar, R. Reese, J.T., Suen, G., Tao, S., Torres, C.W., Tsutsui, N.D., Viljakainen, L., Wolschin, F., Gadau, J., 2011b. Draft genome of the red harvester ant Pogonomyrmex barbatus. Proc. Natl. Acad. Sci. U.S.A. 108, 5667-5672.
Sobolevsky, A.I., Rosconi, M.P., Gouaux, E., 2009. X-ray structure, symmetry and mechanism of an AMPA-subtype glutamate receptor. Nature 462, 745-756.

Stamatakis, A., 2006. RAxML-VI-HPC: maximum likelihood-based phylogenetic analyses with thousands of taxa and mixed models. Bioinformatics 22, 2688-2690. Stepanyan, R., Hollins, B., Brock, S.E., McClintock, T.S., 2004. Primary culture of lobster (Homarus americanus) olfactory sensory neurons. Chem. Senses 29, 179-187.

Su, C.Y., Menuz, K., Carlson, J.R., 2009. Olfactory perception: receptors, cells, and circuits. Cell 139, 45-59.

Ulbrich, M.H., Isacoff, E.Y., 2007. Subunit counting in membrane-bound proteins. Nat. Methods 4, 319-321.

van der Goes van Naters, W., Carlson, J.R., 2006. Insects as chemosensors of humans and crops. Nature 444, 302-307.

Vosshall, L.B., Stocker, R.F., 2007. Molecular architecture of smell and taste in Drosophila. Annu. Rev. Neurosci. 30, 505-533.

Yao, C.A., Ignell, R., Carlson, J.R., 2005. Chemosensory coding by neurons in the coeloconic sensilla of the Drosophila antenna. J. Neurosci. 25, 8359-8367.

Zhan, S., Merlin, C., Boore, J.L., Reppert, S.M., 2011. The monarch butterfly genome yields insights into long-distance migration. Cell 147, 1171-1185.

Zhou, X., Slone, J.D., Rokas, A., Berger, S.L., Liebig, J., Ray, A., Reinberg, D. Zwiebel, L.J., 2012. Phylogenetic and transcriptomic analysis of chemosensory receptors in a pair of divergent ant species reveals sex-specific signatures of odor coding. PLoS Genet. 8, e1002930. 